\title{
Estimation of aggregation kernels based on Laurent polynomial approximation
}

\author{
H. Eisenschmidt ${ }^{1}$, M. Soumaya ${ }^{1}$, N. Bajcinca ${ }^{2,3}$, S. Le Borne ${ }^{4}$, K. Sundmacher ${ }^{1,2^{*}}$ \\ 1 Otto-von-Guericke-University Magdeburg, Department Process Systems \\ Engineering, Universitätsplatz 2, D-39106 Magdeburg, Germany \\ 2 Max Planck Institute for Dynamics of Complex Technical Systems, Department \\ Process Systems Engineering, Sandtorstr.1, D-39106 Magdeburg, Germany \\ 3 University of Kaiserslautern, Department of Mechatronics in Mechanical and Automotive \\ Engineering, Gottlieb-Daimler-Straße 42, D-67663 Kaiserslautern, Gernamy \\ 4 Hamburg University of Technology, Institute for Mathematics, Am Schwarzenberg-Campus \\ 3, 21073 Hamburg, Germany \\ *Corresponding autor: sundmacher@mpi-magdeburg.mpg.de
}

\begin{abstract}
The dynamics of particulate processes can be described by population balance equations which are governed by the phenomena of growth, nucleation, aggregation and breakage. Estimating the kinetics of the latter phenomena is a major challenge particularly for particle aggregation because first principle models are rarely available and the kernel estimation from measured population density data constitutes an ill-conditioned problem. In this work we demonstrate the estimation of aggregation kernels from experimental data using an inverse problem approach. This approach is based on the approximation of the aggregation kernel by use of Laurent polynomials. We show that the aggregation kernel can be well estimated from in silico data and that the estimation results are robust against substantial measurement noise. The method is demonstrated for three different aggregation kernels. Good agreement between true and estimated kernels was found in all investigated cases.
\end{abstract}

\section{Keywords:}

Aggregation, Aggregation kernel, Inverse methods, Polynomial approximation

\section{Introduction}

The phenomenon of aggregation is seen in a variety of different chemical production processes such as droplet coalescence, cell flocculation, granulation and crystal agglomeration. In an aggregation process, two particles with volumes $v$ and $u$ collide and form a new stable particle with the volume $v+u$. The kinetics of such an aggregation process is governed by the aggregation kernel, which is, in the univariate case, a symmetric and nonnegative function that depends on particle volumes $v$ and $\mathrm{u}$ and on further process conditions, like fluid dynamics or concentrations, which can be time-dependent. The aggregation kernel is typically modeled as a product of a collision kernel and an aggregation efficiency (Hounslow et al., 2001; Ochsenbein et al., 2015). Here, the collision kernel is typically assumed to be dependent on the sizes of the aggregating particles as well as on the fluid dynamics of the system. The aggregation efficiency can be interpreted as the probability that a particle collision leads to a stable aggregate. For instance, in crystallization processes solid bridges have to be formed between two colliding primary crystals so that a stable crystal agglomerate is formed (Hounslow et al., 2001; Ochsenbein et al., 2015). This process depends on the particle inertias, and thus on the particle sizes, as well as on process conditions such as 
the supersaturation of the solution surrounding the aggregating particles which can be timedependent.

Once a suitable model for the aggregation process is formulated, the aggregation kernel can be determined by fitting unknown model parameters to the measured transient evolution of the particle size distribution. Examples of this procedure can for instance be found in the field of crystallization (Lindenberg et al., 2008), precipitation (Bramley et al., 1996) or granulation (Peglow et al., 2006; Braumann and Kraft, 2010; Braumann et al., 2011).

If no a priori knowledge of the structure of the aggregation kernel is known, the aggregation kernel can also be obtained from experimental data by the inverse problem approach. An inverse problem arises, when the solution to a partial differential equation, i.e. the population balance equation, is known, i.e. from measurements, while functions governing this solution, in the here discussed case the aggregation kinetics, are unknown (Ramkrishna, 2000). The inverse problem approach to population balance systems requires the observation of the dynamic evolution of the number density evolution, but it does not (necessarily) require any a-priori knowledge about the underlying structure of the kinetic expression to be determined. However, the solution of an inverse problem often results in an ill-conditioned problem (Chakraborty et al., 2015).Mahoney et al. (2002) for instance have demonstrated the solution of inverse problems to determine growth and nucleation rates in a precipitation process from simulated measurements. The estimation of aggregation kernels was demonstrated by Wright and Ramkrishna (1992), based on the assumption of self-similarity. The latter approach was subsequently used by other groups for determining kernels for stem cell aggregation (Rostami et al., 2015) and sludge flocculation (Torfs et al., 2012). The self-similarity of the particle size distribution depends on the aggregation kernel as well as on the initial conditions and may not be achieved under conditions where aggregation and growth occur simultaneously, as for instance reported by Bramley et al. (1997). Therefore, Chakrabortyet al. (2015) have suggested the estimation of the aggregation kernel by use of the method of weighted residuals. This method results in an ill-conditioned system of linear equations which can be solved using regularization techniques.

The present work aims at complementing the approach of Chakraborty et al. (2015) by proposing a kernel estimation scheme based on polynomial approximation which, besides the kernel symmetry, does not rely on any assumption about the structure of the aggregation kernel or the transient evolution of the particle size distribution. The remainder of this article is structured as follows. In Section 2, the approach for the kernel estimation is presented together with the results obtained from datasets without measurement noise. The estimation performance in the presence of measurement noise is presented and discussed in Section 3, where also a statistical analysis of the kernel estimates is presented, to determine the adequate number of terms in the polynomials. Finally, Section 4 concludes the article and gives an outlook on further improvements, challenges and extensions to the multivariate case of the proposed kernel estimation procedure.

\section{Kernel estimation by polynomial approximation}

The evolution of a particle distribution during an aggregation process can be described by a population balance equation (PBE)

$$
\frac{\partial f(v, t)}{\partial t}=\frac{1}{2} \int_{0}^{v} k(v-u, u) f(v-u, t) f(u, t) \mathrm{d} u-\int_{0}^{\infty} k(v, u) f(v, t) f(u, t) \mathrm{d} u,
$$

with the initial condition:

$$
f(v, t=0)=f_{0}(v) .
$$


In both equations, $f$ stands for the number density distribution, and $v$ for the particle volume. The first integral term of the right hand side of Eq. (1) represents the birth of particles with volume $v$, which results from the aggregation of two particles with volumes $u$ and $v-u$, respectively. The second integral term, typically referred to as death term, describes the aggregation of two particles with volumes $v$ and $u$ which are forming a new particle having the volume $v+u$. Hence, this process leads to the disappearance of particles from the size class $v$ and acts as a sink term. For solving the population balance equation, the volume coordinate is discretized on an equidistant grid ranging from $v_{1}=1$ to $v_{N c}=N_{C}$. In this notation, the value of $v_{1}$ corresponds to the minimal size of a primary particle, whereas $N_{C}$ denotes the total number of considered size classes which is set to $N_{\mathrm{C}}=50$ in this work. By integrating Eq. (1) w.r.t. the size domain, a set of differential equations for the total number of particles in the volume class $v_{i}$ is obtained:

$$
\frac{d N_{v_{i}}(t)}{d t}=\frac{1}{2} \sum_{j=1}^{i-1} k\left(v_{i}-u_{j}, u_{j}\right) N_{v_{i}-u_{j}}(t) N_{u_{j}}(t)-\sum_{j=1}^{N_{C}} k\left(v_{i}, u_{j}\right) N_{v_{i}}(t) N_{u_{j}}(t) .
$$

The initial condition that was used throughout this work is given by:

$$
N_{v_{i}}(t=0)=\exp \left(-0.2 v_{i}\right) \text {. }
$$

The dynamics of the aggregation process described by Eq. (3) are governed by the aggregation kernel $k$, which is assumed to be independent of time. The aim of this work is the estimation of this kernel. For developing and assessing the estimation procedure described below, we use three different kernel functions which are given in Table 1 and shown in Figure 1. These kernels are chosen to represent qualitatively different curvatures and dependencies on the particle sizes $v$ and $u$. They include two kernels derived theoretically from Brownian motion and from the kinetic theory (Aldous 1999) and also an empirical kernel describing particle aggregation in a granulation process (Peglow 2006).
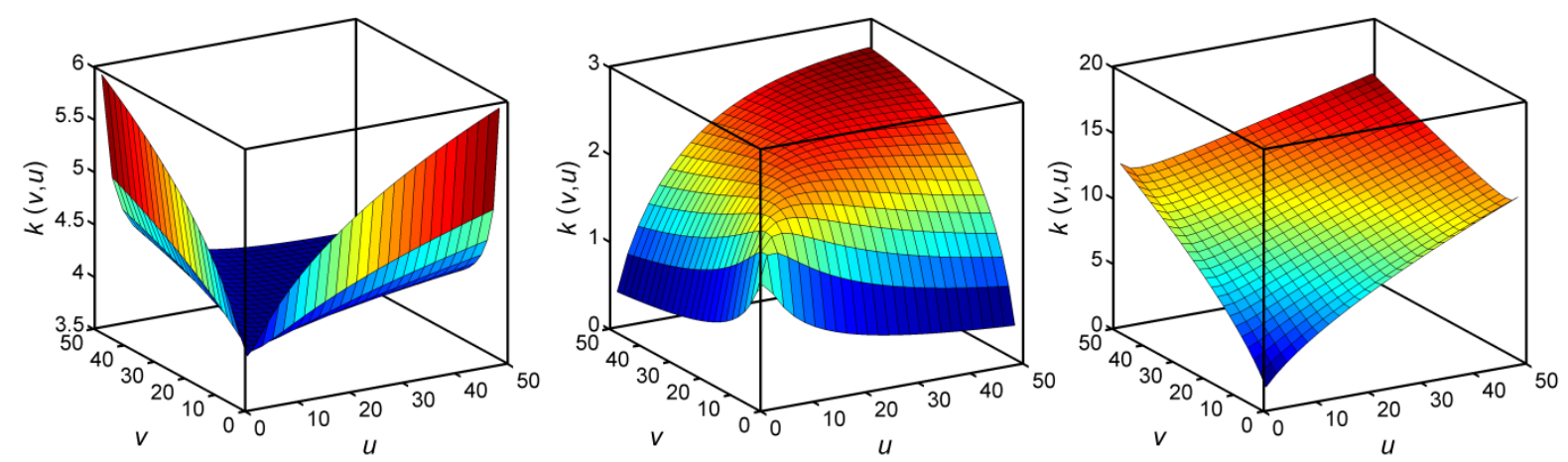

Figure 1: Example aggregation kernels used in this study: left: Brownian kernel; middle: kinetic kernel; right: granulation kernel.

The datasets that are used for the kernel estimation are simulated with these three kernels. Solutions of Eq. (3) are obtained at $N_{t}=50$ linearly spaced time instances between 0 and 1. The numerical solution of the PBE is exemplarily shown in Figure 2 for the kinetic kernel.

Table 1: Example kernels used in this work

\begin{tabular}{|ll|}
\hline Kernel & Expression \\
Brownian kernel (Aldous 1999) & $k(v, u)=\left(v^{1 / 3}+u^{1 / 3}\right)\left(v^{-1 / 3}+u^{-1 / 3}\right)$ \\
kinetic kernel (Aldous 1999) & $k(v, u)=\left(v^{1 / 3}+u^{1 / 3}\right)^{2}(v u)^{1 / 2}(v+u)^{-3 / 2}$ \\
granulation kernel (Peglow 2006) & $k(v, u)=(v+u)^{0.7105}(v u)^{-0.062}$ \\
\hline
\end{tabular}



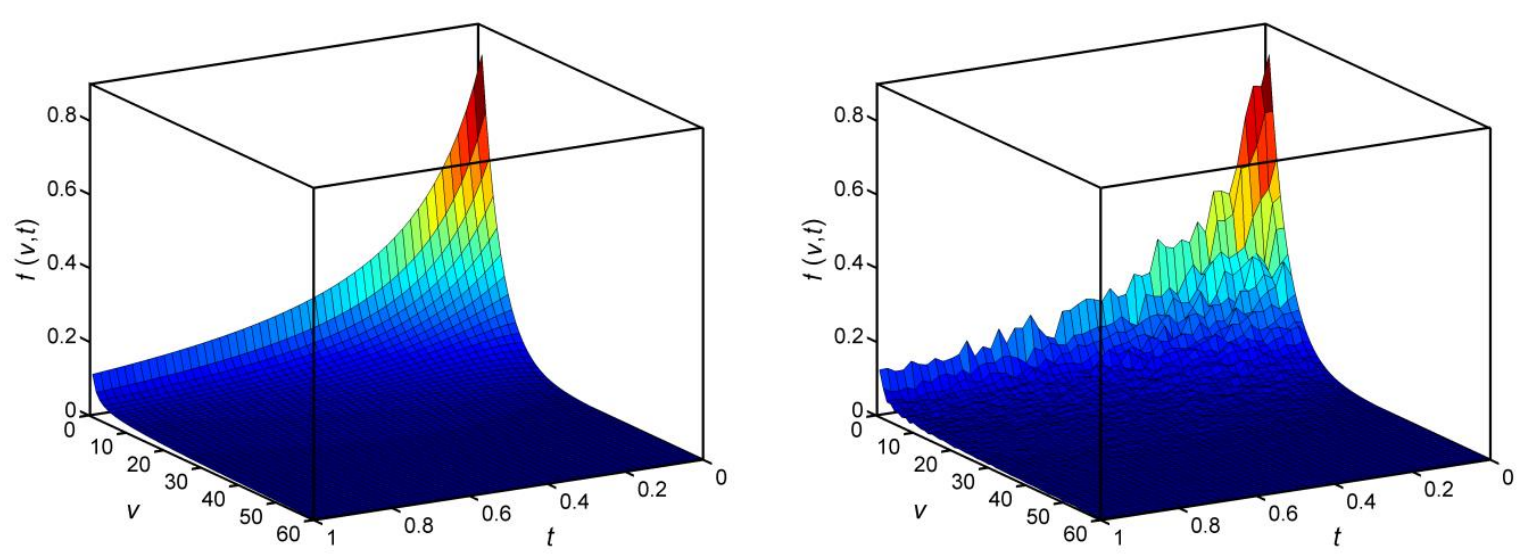

Figure 2: Evolution of the number density distribution for the kinetic kernel; left: without noise; right: with white Gaussian noise and a standard deviation of $10 \%$.

In view of the complexity of Eq. (3) it is clear, that the direct estimation of all kernel function values at every combination of particle volumes $v$ and $u$ is not feasible, as the number of unknown variables scales quadratically with the number of size classes $N_{\mathrm{C}}$ considered. Hence, the kernel must be approximated by a suitable set of basis functions. In this work, Laurent polynomials are chosen for representing the kernel functions:

$$
k_{\text {est }}(v, u)=\sum_{m=-R}^{R} \sum_{n=-R}^{R} p_{m, n} v^{m} u^{n} .
$$

Eq. (5) constitutes a separable approximation of the aggregation kernel, and hence, the source term of Eq. (3) can be evaluated efficiently through fast Fourier transformation (Hackbusch 2006, Le Borne 2015) which becomes significant for larger number of size classes $N_{\mathrm{C}}$ ). Imposing symmetry on the approximated aggregation kernel and ordering the individual terms in an increasing order, the polynomial kernel approximation using rank $R=2$, which is used throughout this work, is given by:

Eq. (5) constitutes a separable approximation of the aggregation kernel, and hence, the source term of Eq. (3) can be evaluated efficiently through fast Fourier transformation (Hackbusch 2006; Le Borne et al., 2015) which becomes significant for larger number of size classes $N_{\mathrm{C}}$. Imposing symmetry on the approximated aggregation kernel and ordering the individual terms in an increasing order, the polynomial kernel approximation using rank $R=2$, which is used throughout this work, is given by:

$$
\begin{aligned}
k_{\text {est }}(v, u)= & p_{1}+p_{2}(v+u)+p_{3}\left(v^{-1}+u^{-1}\right)+p_{4}(v \cdot u)+p_{5}\left(v^{-1} u+u^{-1} v\right)+\ldots \\
& p_{6}\left(v^{-1} u^{-1}\right)+p_{7}\left(v^{2}+u^{2}\right)+p_{8}\left(v^{-2}+u^{-2}\right)+p_{9}\left(v^{2} u+u^{2} v\right)+\ldots \\
& p_{10}\left(v^{-2} u+u^{-2} v\right)+p_{11}\left(v^{-1} u^{2}+u^{-1} v^{2}\right)+p_{12}\left(v^{-2} u^{-1}+u^{-2} v^{-1}\right)+\ldots \\
& p_{13}\left(v^{2} u^{2}\right)+p_{14}\left(v^{-2} u^{2}+u^{-2} v^{2}\right)+p_{15}\left(v^{-2} u^{-2}\right)
\end{aligned} .
$$

Here, the parameters $p_{1}$ to $p_{15}$ are the unknown coefficients of the kernel which have to be determined from the observed evolution of the particle size distribution. Due to the fact, that the right hand side of the differential Eq. (3) is linear with respect to the aggregation kernel, which in turn is linear w.r.t. the unknown coefficients $p_{1}$ to $p_{15}$, a linear regression problem can be formulated for estimating these coefficients, that minimizes the deviations between the measured derivatives $d N_{v, i, \text { meas }} / d t$ and simulated values of the derivatives $d N_{v, i \text {,est }} / d t$ for all size classes and observation times. By collecting all unknown coefficients $p_{1}$ to $p_{15}$ in the parameter vector $\mathrm{p}$, this linear regression problem can be formulated as follows: 


$$
\min _{\mathbf{p}} \sum_{i=1}^{N_{C}} \sum_{j=1}^{N_{t}}\left(\frac{d N_{v_{i}, \text { meas }}\left(t_{j}\right)}{d t}-\frac{d N_{v_{i}, \text { est }}\left(t_{j}, \mathbf{p}\right)}{d t}\right)^{2} .
$$

Although this problem can be solved analytically, it requires the derivatives $d N_{v, i \text {, meas }} / d t$ and may suffer from an ill-conditioned estimation problem (Chakraborty et al., 2015). For these reasons, instead of solving the optimization problem directly, we apply an iterative optimization procedure in which the number of optimized parameters is successively increased by one and the optimal values of the previous iteration are used for initialization. Further-more, we scale the derivatives $d N_{v, l} / d t$ of all size classes by the observed maximal particle number in each class, in order to account for the different orders of magnitude of the particle numbers, see Figs 2 and 5. Thereby, the sensitivity of the objective function w.r.t. the kernel function values at combinations of large particle sizes $v$ and $u$ is increased significantly:

$$
\min _{\mathbf{p}} \sum_{i=1}^{N_{C}} \frac{1}{\max N_{v_{i}, \text { meas }}} \sum_{j=1}^{N_{t}}\left(\frac{d N_{v_{i}, \text { meas }}\left(t_{j}\right)}{d t}-\frac{d N_{v_{i}, \text { est }}\left(t_{j}, \mathbf{p}\right)}{d t}\right)^{2} .
$$

In this approach, the derivatives $d N_{v, i, \text { meas }} / d t$ have to be known and thus must be determined from the observed particle number evolution $N_{v, i}(t)$, which is a challenging task in the presence of measurement noise as discussed in Section 3. As an alternative objective function, the sum of squares of the differences between measured and simulated number densities $N_{v, i \text {, meas }}$ and $N_{v, i, \text { est }}$ is minimized:

$$
\min _{\mathbf{p}} \sum_{i=1}^{N_{C}} \frac{1}{\max N_{v_{i}, \text { meas }}} \sum_{j=1}^{N_{t}}\left(N_{v_{i} \text {,meas }}\left(t_{j}\right)-N_{v_{i}, \text { est }}\left(t_{j}, \mathbf{p}\right)\right)^{2} .
$$

Again, the measured and simulated values of $N_{v, i}(t)$, are scaled by the maximum particle numbers which are observed in the size class $v_{i}$ to increase the sensitivity of the objective function w.r.t. the kernel function values at combinations of large particle sizes $v$ and $u$. Clearly, the estimated number density evolutions $N_{v, i, \text { est }}(t, \mathbf{p})$ can be obtained by integrating Eq. (3) for a given aggregation kernel which is parameterized by p. However, this significantly increases the computational cost of this approach, since a system of ordinary differential equations (Eq. (3)) has to be solved during every evaluation of the objective function Eq. (9). In this work, both optimization problems are implemented in Matlab 2010b, and solved numerically by the Marquardt-Levenberg algorithm provided by the routine lsqnonlin of the optimization toolbox (version 5.1).
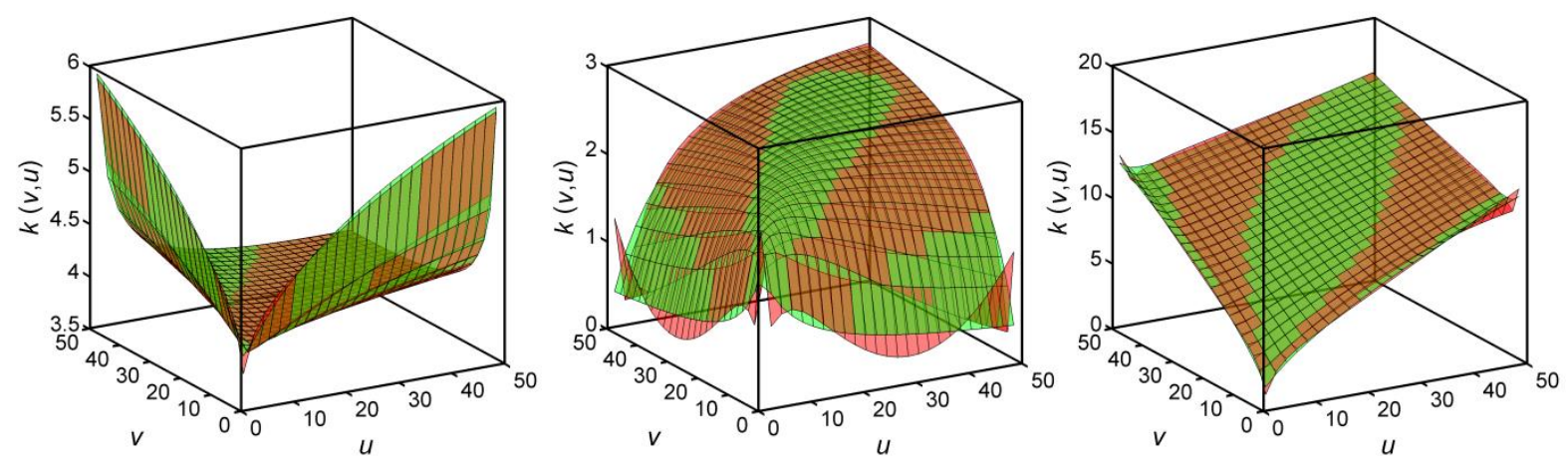

Figure 3: Comparison between the true kernel functions (green) and kernel functions estimated from perfect synthetic data (red); left: Brownian kernel; middle: kinetic kernel; right: granulation kernel. 

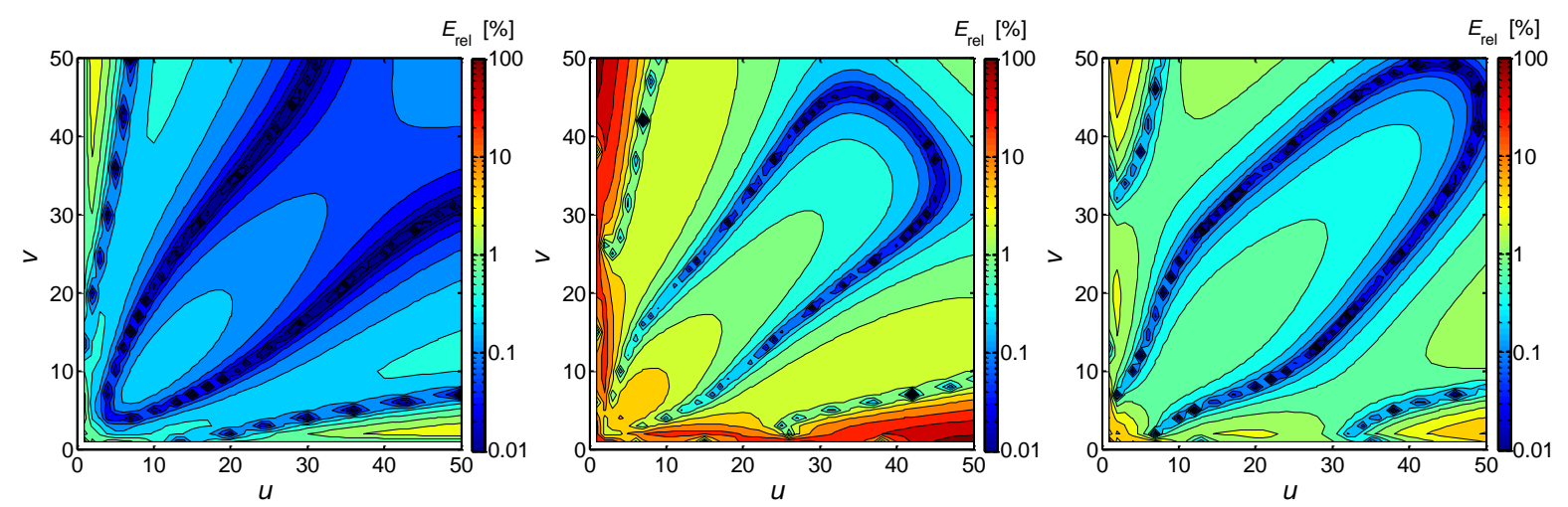

Figure 4: Estimation error from perfect synthetic data; left: Brownian kernel; middle: kinetic kernel; right: granulation kernel.

The estimates of the three test kernel functions that are obtained by the presented approach are shown in Fig. 3 together with the true kernel functions. As both objectives, Eq. (8) and Eq. (9), result in almost identical kernel estimates, only the results of Eq. (8) are shown. As can be seen, the Brownian aggregation kernel and the granulation kernel can be reconstructed almost exactly. Only for the kinetic kernel some larger differences between true and estimated kernel functions are visible. For a quantitative analysis of the estimation performance, the relative estimation error

$$
E_{\text {rel }}=100\left|\frac{k_{\text {true }}-k_{\text {est }}}{k_{\text {true }}}\right|
$$

is shown in Fig. 4 for all three test kernels. It can be seen, that the estimation error is below $1 \%$ for the Brownian kernel and below $2 \%$ for the kinetic and granulation kernel in a wide range of particle size combinations. Only for size combinations that represent the agglomeration of small and large sized particles some larger deviations are visible which is particularly true for the kinetic kernel, where the estimation error reaches values of up to $60 \%$, see also Fig. 3. This indicates that the sensitivity of the objective functions w.r.t. the kernel function values at these size combinations is comparably low. This phenomenon is discussed in the next section, where the influence of the measurement noise on the performance of the estimation scheme is addressed.

\section{Kernel estimation in the presence on measurement noise}

The estimation of aggregation kernels from perfect datasets constitutes a rather idealized case. In reality, the measurements of the number density evolution will be affected by measurement noise. In particular, the derivatives of the number density with respect to time will generally be unknown, which prohibits the direct application of the objective function Eq. (8) for the kernel estimation. For assessing the applicability and performance of the proposed estimation scheme, we simulate the dynamic evolution of the number density for all three test kernels and add white Gaussian noise with a standard deviation of 10 percent of the actual simulated values to the datasets. The resulting number density evolutions are exemplarily shown in Figs. 2 and 5 for the kinetic kernel and the Brownian kernel, respectively.

Of course, a direct estimation of the derivatives $d N_{v, i} / d t$ from such datasets by finite differences is not possible. An approach for the estimation of derivatives of the solution of Eq. (3) is for example given by Vikhansky and Kraft (2004). In this work, however, we estimate the derivatives based on smoothed measurements $N_{v, i}$, which enables the estimation of the derivatives based on the smoothened curves. For this purpose, an extended Kalman filter (Brown and Hwang, 1995) is applied, which exploits the knowledge, that the true number 
density evolution should satisfy Eq. (3). Furthermore, the Kalman filter uses the estimates of the aggregation kernel which are already available after the first iteration. The resulting filtered number density evolution is shown in Fig. 5 together with the measurements and the original noise-free number density evolution. As can be seen, the Kalman filter allows for an almost exact reconstruction of the original true dataset for all size classes, and results in excellent smoothness of the estimates. This allows the direct estimation of the derivatives $d N_{v, i} / d t$ via finite differences. To this end, we choose the following second order method (Ahnert and Abel,2007):

$$
\frac{d N_{v_{i} \text {,est }}\left(t_{j}\right)}{d t}=\frac{4}{3} \frac{N_{v_{i}}\left(t_{j}+\Delta t\right)-N_{v_{i}}\left(t_{j}-\Delta t\right)}{2 \Delta t}-\frac{1}{3} \frac{N_{v_{i}}\left(t_{j}+2 \Delta t\right)-N_{v_{i}}\left(t_{j}-2 \Delta t\right)}{4 \Delta t} .
$$

The resulting estimates for the derivatives are depicted in the right part of Fig. 5. As can be seen, the estimated derivatives match the true values reasonably well, despite some deviation at early simulation time steps and small oscillations during the aggregation process. Clearly, the application of an additional filtering step on the estimated derivatives might lead to an even better estimation quality, especially at later simulation time steps. This approach is, however, not further pursued in this work. Instead, the filtered values $N_{v, i}$ and $d N_{v, i} / d t$ that are obtained from the kernel estimates from the previous iteration are used directly for the kernel estimation. Since no kernel estimate is available at the first iteration, the measurements $N_{v, i \text { meas }}$ are smoothed by a $7^{\text {th }}$ order polynomial, and the derivatives are obtained from these polynomials.
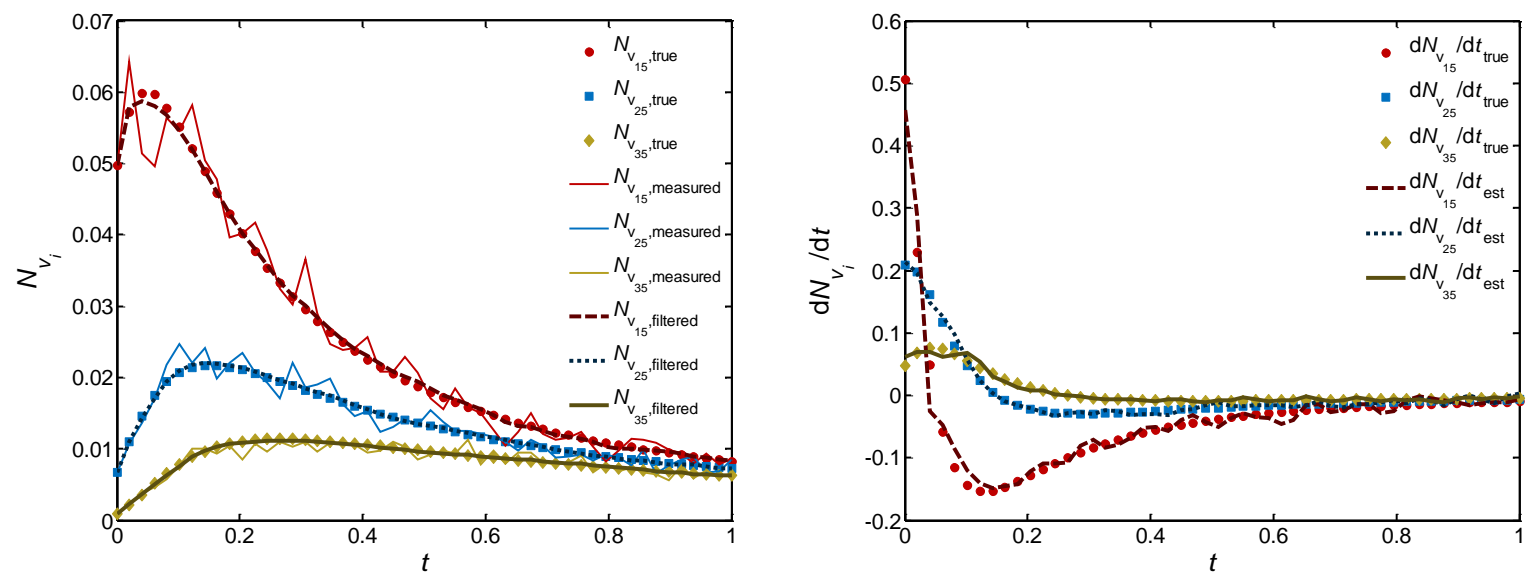

Figure 5: Application of a Kalman filter to noisy data simulated with the brownian kernel; left: true, noisy and filtered evolution of three selected size classes; right: true and estimated derivatives of the same size classes.

The application of a full second order Laurent polynomial (Eq.(6)) for the estimation of the aggregation kernels necessitates the determination of 15 parameters in total. While these parameters can be determined well from perfect datasets (see Figs. 3 and 4), the estimation of these parameters from noisy datasets will most likely result in overfitting of the measurement data, and hence lead to rather poor estimation results. This effect is shown in Fig. 6, where the relative root-mean-square error

$$
\mathrm{RMSE}_{\mathrm{rel}}=\frac{100}{N_{C}} \sqrt{\sum_{i=1}^{N_{C}} \sum_{j=1}^{N_{C}}\left(\frac{k_{\text {true }}\left(v_{i}, u_{j}\right)-k_{\text {est }}\left(v_{i}, u_{j}\right)}{k_{\text {true }}\left(v_{i}, u_{j}\right)}\right)^{2}}
$$

is plotted against the number of parameters used for the estimation of the Brownian aggregation kernel. The relative error decreases initially to reach a minimum when the first five parameters are used for kernel estimation. Afterwards, the relative error increases 
together with the standard deviation of the estimation error with an increase of the number of estimated parameters.

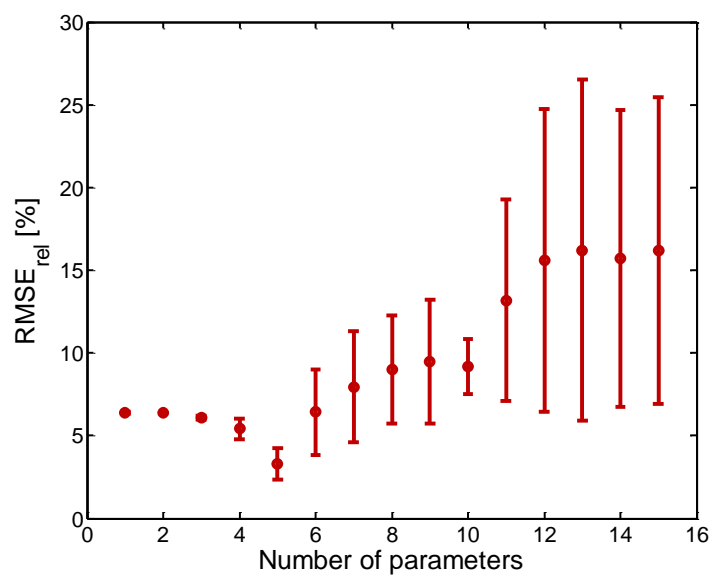

Figure 6: Dependence of the relative root-mean-square error, $\mathrm{RMSE}_{\mathrm{rel}}$, on the number of parameters used for the estimation of the brownian aggregation kernel. The error bars represent the standard deviations obtained from five different random datasets.
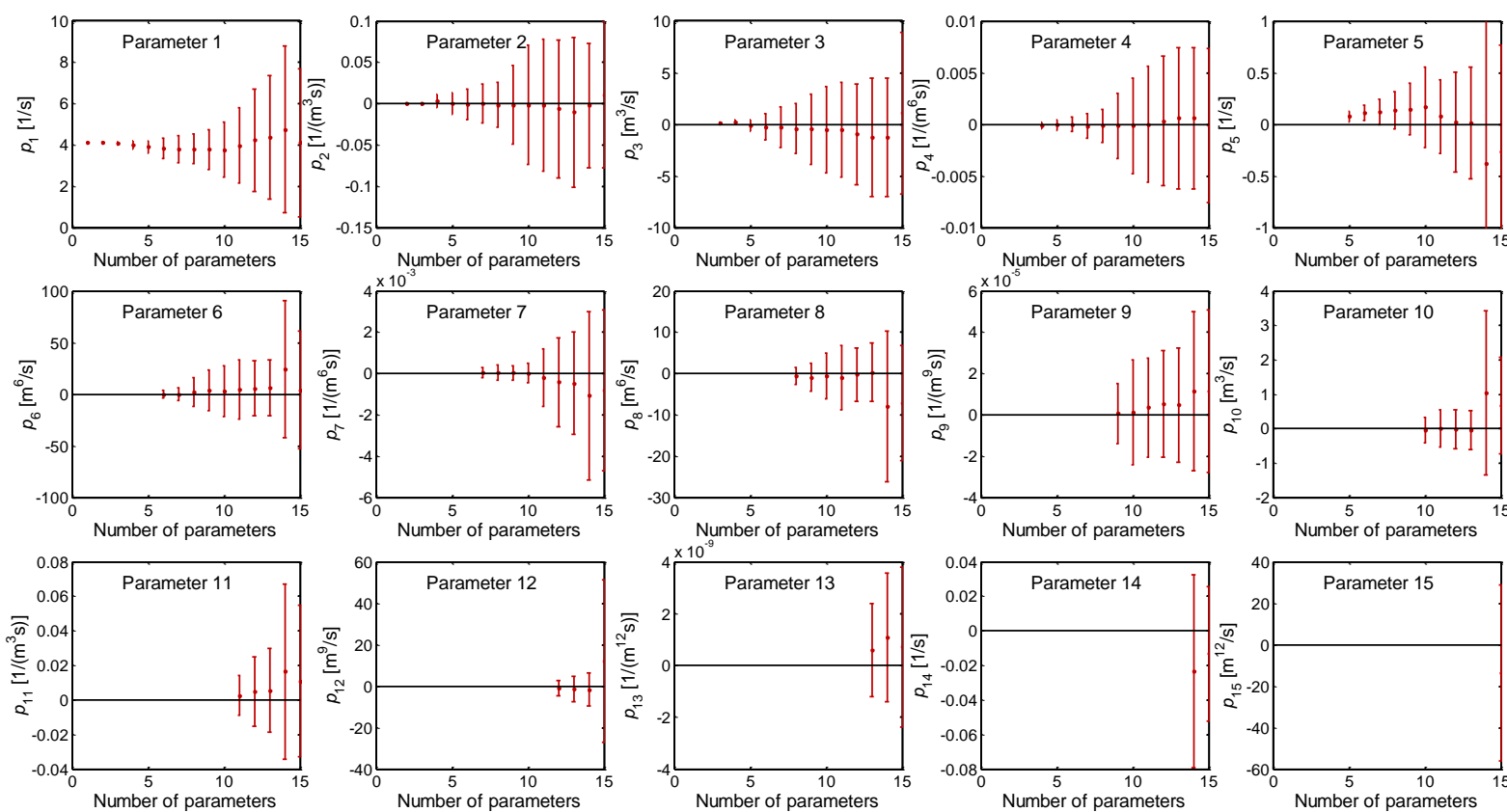

Figure 7: 95\% confidence intervals of the individual parameter estimates for all polynomial sizes considered. Data were simulated with the brownian aggregation kernel, and five independent measurement sets were used to derive the confidence intervals.

Since the true aggregation kernel is unknown, such analysis can of course not be used to determine the number of parameters which are necessary to estimate the aggregation kernel. Instead, five independent measurement sets are used for each test kernel to obtain five sets of independently estimated parameters. Due to the iterative estimation procedure described above, these parameter sets contain parameter estimates for all polynomial sizes containing 115 parameters. By this procedure, confidence intervals for the individual parameter values are obtained from the repeated estimations for every polynomial size. The results of this analysis are shown for the example of the Brownian aggregation kernel in Fig. 7 for a significance level of $95 \%$. As can be seen, the confidence intervals for essentially all parameters are increasing at increasing number of estimated parameters, which can be attributed to parameter correlation. In fact, with all 15 parameters estimated, only the first parameters (top left) 
significantly deviate from zero. For determining an adequate polynomial basis, we use the polynomial with the maximal number of parameters, at which the estimate of the last parameter significantly deviates from zero. For the kernel example shown in Fig. 7, this is the case for a polynomial with five parameters, see first error bar in the top right subfigure, which corresponds well with the analysis of the relative root-mean-square error shown in Fig. 6. In principle, this significance analysis can be even extended to account for the fact that the order of the terms in Eq. (6) can be changed which would result in a combinatorial problem, and is - for the sake of simplicity - not considered in this work. After determining the number of parameters, the aggregation kernel is estimated on the basis of all five individual measurement sets.
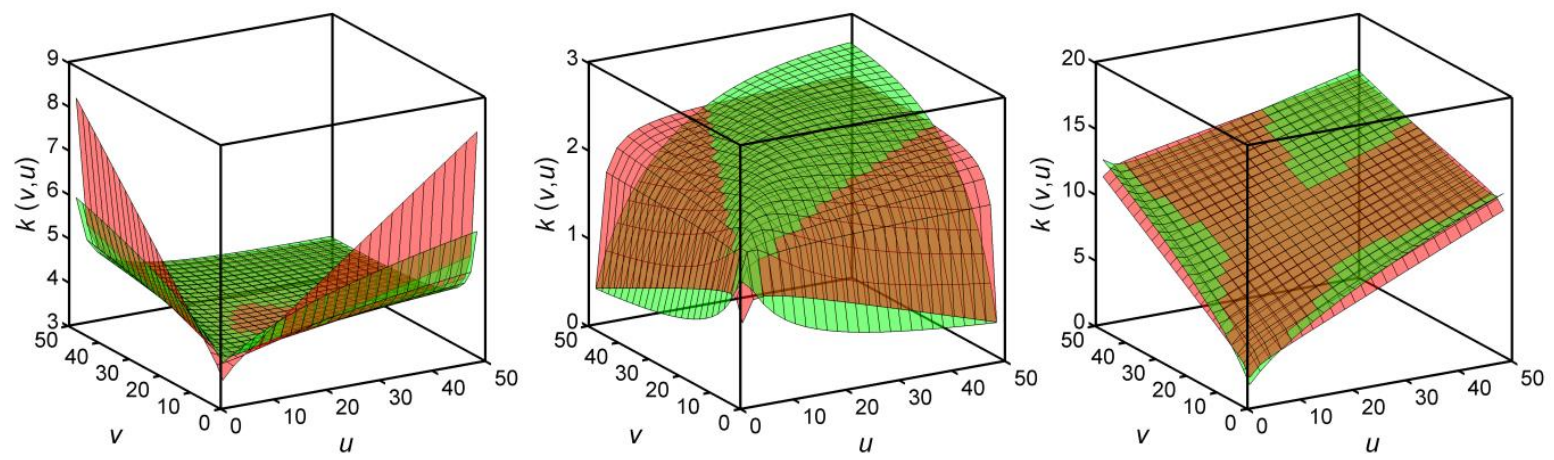

Figure 8: Comparison between the true kernel functions (green) and kernel functions estimated from noisy measurement data using Eq. (9) (red); left: Brownian kernel; middle: kinetic kernel; right: granulation kernel.
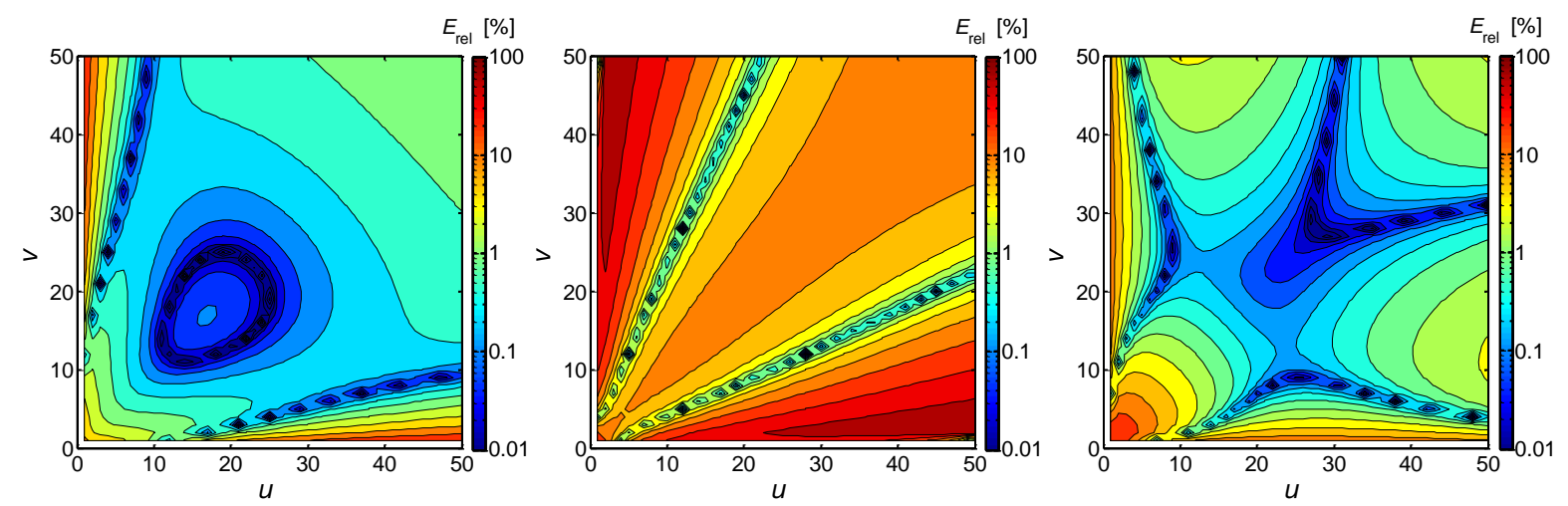

Figure 9: Estimation error from noisy measurement data using Eq. (9); left: Brownian kernel; middle: kinetic kernel; right: granulation kernel.

The final estimates for the three test kernels which are resulting from the application of objective function of Eq. (9) are shown in Fig. 8 together with the true aggregation kernels. The corresponding estimation errors of Eq. (10) are depicted in Fig. 9. The results of the application of Eq. (8) as objective function are shown in Figs. 10 and 11 respectively. In case of the Brownian kernel, a polynomial with five parameters is used for the final kernel estimation for both objective functions. As can be seen, the kernel is estimated reasonably well, exhibiting a characteristic plateau at high values of $\mathrm{u}$ and $v$, were the estimation error is in the range of $1-2 \%$. However, the kernel function values that describe the interactions between small and large sized particles are overestimated by both approaches, resulting in rather high estimation errors. Again, this indicates a rather low sensitivity of the objectives to kernel function values in this area, as it was already observable in the estimation from perfect datasets, particularly for the kinetic kernel and partially for the granulation kernel (see Figs. 3 and 4).The low sensitivity is due to the low probability of small and large sized particles 
coexisting, and hence aggregating, at the same time. This is a direct consequence of the initial conditions chosen for simulation as well as of the aggregation process itself. A higher sensitivity in this region of the kernel function can be obtained either by using an optimized initial condition or by addition of a second particle population during the aggregation process. Both approaches would increase the probability of the coexistence of small and large size particles, but are not further elaborated in the present study.

The estimation performance of the proposed scheme is some-what lower for the kinetic kernel than for the Brownian kernel. Although the general concave shape of the kernel function can be restored with both approaches, systematic deviations in the range of $10 \%$ to $70 \%$ between true and estimated kernel functions are visible for various size combinations. Both estimated kernel functions have a rather similar shape, which indicates, that the polynomial with five parameters that is chosen in both cases might not be flexible enough to reproduce the true kernel function with sufficient accuracy. Polynomials of higher order are, however, dropped by the above described test.

The estimated granulation kernel, obtained by Eq. (9), almost exactly matches the true kernel for almost all considered size combinations, while the application of Eq. (8) leads to some slight deviations which can, however, still be considered as acceptable, as the estimation error does not exceed 10-20\%. The reason for this behavior is the different number of parameters used for the kernel estimations. While four parameters were found to be significant incase of Eq. (9), only the first three parameters were used for the kernel estimation by Eq. (8), which explains the observed deviations between the estimates in Figs. 8 and 10.
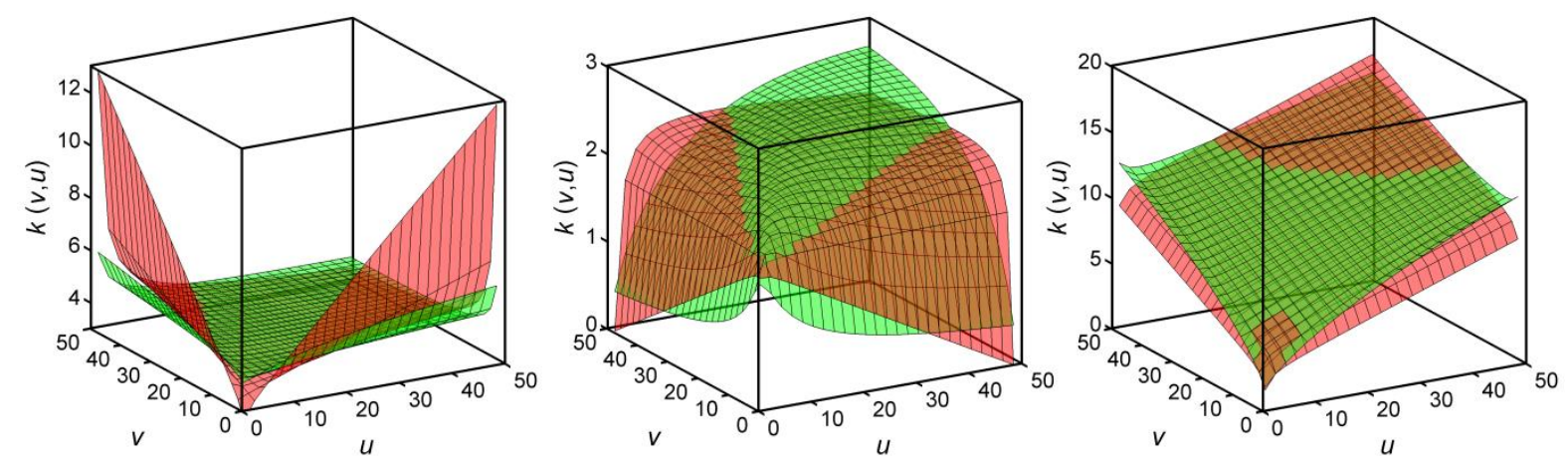

Figure 10: Comparison between the true kernel functions (green) and kernel functions estimated from noisy measurement data using Eq. (7) (red); left: brownian kernel; middle: kinetic kernel; right: granulation kernel.
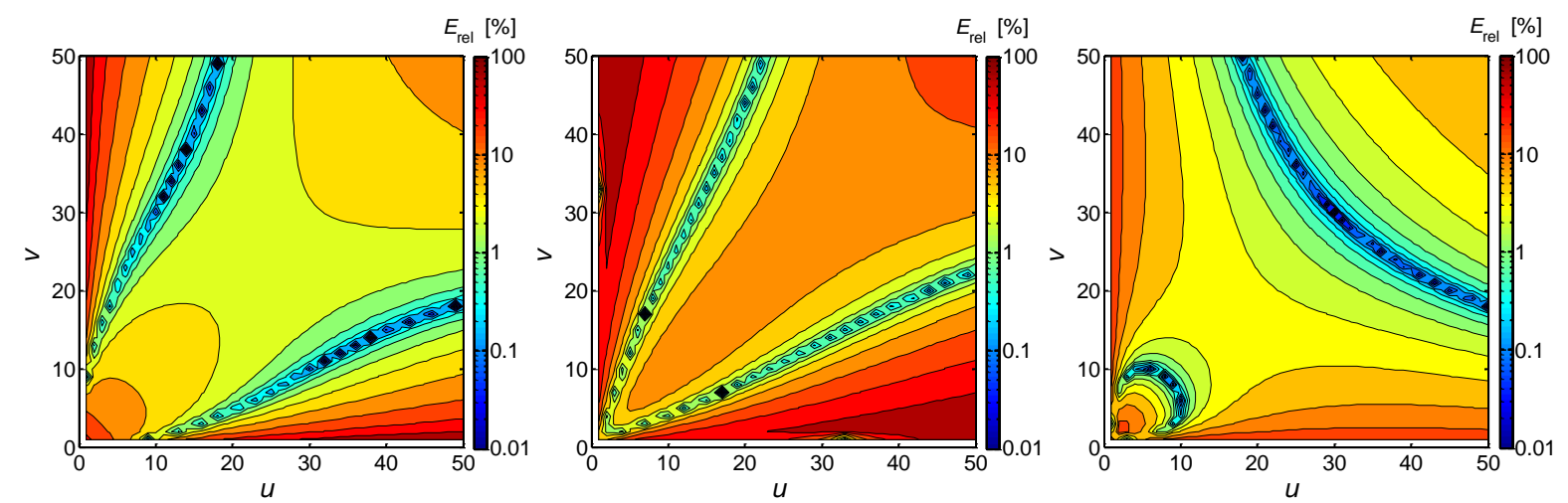

Figure 11: Estimation error from noisy measurement data using Eq. (7); left: brownian kernel; middle: kinetic kernel; right: granulation kernel. 


\section{Conclusions and Outlook}

In this work, we demonstrated a method for the estimation of aggregation kernels based on observations of the evolution of the particle size distribution over time. Due to the polynomial approximation of the kernel, no a priori knowledge about the nature of this kernel is required, and hence, this method might be applicable in a wide range of aggregation processes. However, this method requires that the true aggregation kernel can be well approximated with the chosen polynomial expression. The example of the kinetic kernel demonstrates that the quality of approximation is in fact dependent on the shape of the original aggregation kernel. To improve the estimation quality in such cases, further polynomial approximations can be considered that for example account for fractional exponents in the volume domain, like 1/3 or $2 / 3$, correlating to the particle length and surface area, which are frequently found in theoretical kernel expressions.

The obtained results demonstrate the applicability of the pro-posed scheme also in the presence of substantial measurement noise. A careful statistical analysis is required to identify a suitable polynomial expression. This analysis requires the availability of either repeated experiments or measurements with a high temporal resolution to facilitate independent sets of parameter estimates. In this respect, some further investigations might be beneficial to give some guidelines about the minimal amount of data required for reliable kernel estimation to reduce the experimental effort. Alternatively, approaches for the simultaneous estimation of the parameters and their confidence intervals as for example proposed by Braumann and Kraft (2010) and Braumann et al. (2011) or Kastner et al. (2013) can be beneficial for the statistical analysis. As the parameter confidence intervals and possible correlations between these parameters are known from this procedure, an adequate polynomial expression could be identified from such an approach. The knowledge of the parameter confidence intervals might also be exploited for the optimal design of the experiments, as for instance demonstrated by Mosbach et al. (2012).

The obtained results indicate that the sensitivity of the objective functions on the kernel function values decreases at combinations of large and small sized particle aggregations. This gives rise to further improvements of the methods, like the choice of initial conditions as well the investigation of optimized seeding strategies. In particular the latter strategy appears to be a promising candidate, if also growth, and hence an additional mechanism for size enlargement, has to be considered. Due to particle growth, a situation might arise, in which particles with a small size have already grown to larger sizes while particles with large sizes had no time to form yet. Hence, a reliable estimation of aggregation frequencies is in this case not possible for these size combinations. The addition of a second seed population at an appropriate time will, however, resolve this problem, as the simultaneous presence of large and small sized particles can be ensured by this approach.

The methods presented in this work are exemplified on the case of univariate particle size distributions. Nevertheless, an extension of the methods to a multivariate particle size distribution is possible. For this purpose, the formulation of the Laurent polynomials (Eq. (5)) has to be extended to account for the additional property coordinates. Provided that the source and sink terms are linearly dependent on the multivariate kernel function, both approaches of Eqs. (8) and (9) can be applied, since the kernel approximation will retain a linear dependence on the coefficients of the polynomial. The extension of the methods to a multivariate case does however require the availability of online measurements of the evolution of the particle size distribution in the multivariate property space. This information might in practice be hard to acquire. Additionally, the extension of the polynomial approach will lead to a significant increase in the number of unknown parameters which have to be estimated, which in turn will give rise to a significant increase in the computational costs of the presented methods. 
This work is exclusively focused on the estimation of aggregation kernels which are independent of time. This assumption might, however, not necessarily be fulfilled as process conditions, which are influencing the aggregation kinetics, might change over time. In this case, a suitable extension of the polynomial approach has to be found which is able to capture the time dependence of the kernel explicitly or implicitly.

\section{Acknowledgements}

The authors gratefully acknowledge the financial support of this research work by the Deutsche Forschungsgemeinschaft (DFG) under the Grants SU 189/6-2 and BO 4141/1-2 in the framework of the priority program SPP 1679 "Dynamische Simulation vernetzter Feststoffprozesse".

\section{References}

Ahnert, K., Abel, M., 2007. Numerical differentiation of experimental data: local versus global methods. Comput. Phys. Commun. 177, 764-774.

Aldous, D.J, 1999. Deterministic and stochastic models for coalescence (aggregation and coagulation): a review of the mean-field theory for probabilists. Bernoulli 5, 3-48.

Bramley, A.S., Hounslow, M.J., Ryall, R.L., 1996. Aggregation during precipitation from solution: A method for extracting rates from experimental data. J. Colloid Interface Sci. $183,155-165$.

Bramley, A.S., Hounslow, M.J., Ryall, R.L., 1997. Aggregation during precipitation from solution. Kinetics for calcium oxalate monohydrate. Chem. Eng. Sci. 52, 747-757.

Braumann, A., Kraft, M., 2010. Incorporating uncertainties into multivariate granulation modeling. Chem. Eng. Sci. 65, 1088-1100.

Braumann, A., Man, P.L.W., Kraft, M., 2011. The inverse problem in granulation modeling two different statistical approaches. AIChE J. 57, 3105-3121.

Brown, R.G., Hwang, P.Y.C., 1995, Introduction to Random Signal Analysis and Kalman Filtering, second ed. John Wiley \& Sons Inc, New York / Chichester / Brisbane / Toronto / Singapore.

Chakraborty, J., Kumar, J., Singh, M., Mahoney, A., Ramkrishna, D., 2015. Inverse problems in population balances. Determination of aggregation kernel by weighted residuals. Ind. Eng. Chem. Res. 54, 10530-10538.

Hackbusch, W., 2006. On the efficient evaluation of coalescence integrals in population balance models. Computing 78, 145-159.

Hounslow, M.J., Mumtaz, H.S., Collier, A.P., Barrick, J.P., Bramley, A.S., 2001. A micromechanical model for the rate of aggregation during precipitation from solution. Chem. Eng. Sci. 56, 2543-2552.

Kastner, C.A., Braumann, A., Man, P.L.W., Mosbach, S., Brownbridge, G.P.E., Akroyd, J., Kraft, M., Himawan, C., 2013. Bayesian parameter estimation for a jet-milling model using Metropolis-Hastings and Wang-Landau sampling. Chem. Eng. Sci. 89, 244-257.

Le Borne, S., Shahmuradyan, L., Sundmacher, K., 2015. Fast evaluation of univariate aggregation integrals on equidistant grids. Comput. Chem. Eng. 74, 115-127.

Lindenberg, C., Schöll, J., Vicum, L., Mazzotti, M., Brozio, J., 2008. L-glutamic acid precipitation: Agglomeration effects. Cryst. Growth Des. 8, 224-237.

Mahoney, A.W., Doyle, F.J., Ramkrishna, D., 2002. Inverse problems in population balances: growth and nucleation from dynamic data. AIChE J. 48, 981-990. 
Mosbach, S., Braumann, A., Man, P.L.W., Kaster, C.A., Brownbridge, G.P.E., Kraft, M., 2012. Iterative improvement of Bayesian parameter estimates for an engine model by means of experimental design. Combust. Flame 159, 1303-1313.

Torfs, E., Dutta, A., Nopens, I., 2012. Investigating kernel structures for Ca-induced activated sludge aggregation using an inverse problem methodology. Chem. Eng. Sci. 70, 176-187.

Ochsenbein, D.R., Vetter, T., Morari, M., Mazzotti, M., 2015. Agglomeration of needle-like crystals in suspension. II. Modeling. Cryst. Growth Des. 15, 4296-4310.

Peglow, M., Kumar, J., Warnecke, G., Heinrich, S., Mörl, L., 2006. A new technique to determine rate constants for growth and agglomeration with size- and time-dependent nuclei formation. Chem. Eng. Sci. 61, 282-292.

Ramkrishna, D., 2000, Population balances: Theory and applications to particulate systems in engineering. Academic Press, San Diego / San Francisco /New York / Boston / London / Sydney / Tokyo.

Rostami, M.R., Wu, J., Tzanakakis, E.S., 2015. Inverse problem analysis of pluripotent stem cell aggregation dynamics in stirred-suspension cultures. J. Biotechnol. 208, 70-79.

Vikhansky, A., Kraft, M., 2004. A Monte Carlo methods for the identification and sensitivity analysis of coagulation processes. J. Comput. Phys. 200, 50-59.

Wright, H. Ramkrishna, D., 1992. Solutions of inverse problems in population balances-I. Aggregation kinetics. Comput. Chem. Eng. 16, 1019-1038. 